\title{
Ideal cardiovascular health and the subclinical impairments of cardiovascular diseases: a cross-sectional study in central south China
}

Ya-Qin Wang ${ }^{1}$, Chang-Fa Wang ${ }^{2}$, Ling Zhu' ${ }^{1}$, Hong Yuan ${ }^{3}$, Liu-Xin Wu ${ }^{4}$ and Zhi-Heng Chen ${ }^{1 *}$

\begin{abstract}
Background: Ideal cardiovascular health $(\mathrm{CVH})$ is related to a low cardiovascular disease risk profile. This study aimed to investigate $\mathrm{CVH}$ metrics with both the biomarkers and markers of subclinical impairments of cardiovascular diseases (CVDs) in subjects from central south China.

Methods: The ideal CVH score (non-smoking status; ideal body mass index; regular physical activity; healthy diet; and an optimal serum cholesterol, blood pressure, and glucose profile; 1 point for each; total score: 0-7) was collected from 3009 participants without a history of CVDs. Subclinical biomarkers were assessed using C-reactive protein, homocysteine, and microalbuminuria. The presence of subclinical disease markers was defined as having at least one of the following: increased carotid intima-media thickness, carotid plaque, left ventricular hypertrophy, left ventricular systolic dysfunction, or a reduced ankle-brachial index. The association of biomarkers and markers with the $\mathrm{CVH}$ score was evaluated using multivariate logistic regression and linear regression analyses.
\end{abstract}

Results: Only $0.2 \%$ of the study participants met all 7 ideal $\mathrm{CVH}$ metrics $(\mathrm{CVH}$ score $=7$ ). Compared to the female participants, the male participants had poorer CVH profiles and a higher incidence of subclinical lesions $(P<0.05)$. In the fully adjusted models, per 1-unit increase in the CVH score was inversely associated with the biomarker levels ( $\beta=-0.092--0.224, P<0.05$ for all) and the odds of the presence of markers (odds ratio, $0.808 ; 95 \%$ confidence interval, 0.755-0.865). Similar relationships were observed in the gender subgroups and were stronger in the females.

Conclusion: A clear inverse association was observed between the biomarkers or markers of subclinical impairments and the $\mathrm{CVH}$ score in a central south Chinese population, implying the importance of ideal $\mathrm{CVH}$ for the primordial prevention of CVDs.

Keywords: Ideal cardiovascular health, Cardiovascular diseases, Subclinical atherosclerosis

\section{Background}

With urbanization, rapid economic development, and an ageing population, cardiovascular diseases (CVDs) have become the leading causes of morbidity and mortality in China, and the burden of these diseases is increasing at an alarming rate [1]. Consequently, a public health policy of primordial prevention has been advocated. CVDs are long-term chronic diseases that develop over

\footnotetext{
* Correspondence: wdove.bb@163.com

'Department of Health Management Centre, the Third Xiangya Hospital, Central South University, Tongzipo Road 138, Changsha, Hunan Province 410013, China

Full list of author information is available at the end of the article
}

decades from risk factors to subclinical impairment and then progress to clinical events [2]. Subclinical impairment, which is the presymptomatic stage of CVDs, is a fundamental precursor that can help identify early subjects who are at high risk for future CVDs and who can benefit the most from early interventions. This approach is also part of primary prevention. Some non-invasive established biomarkers and markers can detect and measure the subclinical stages of CVDs, such as Creactive protein $(\mathrm{CRP})$, carotid intima-media thickness (CIMT), carotid plaque, left ventricular (LV) hypertrophy, and LV systolic dysfunction. 
In 2010, the American Heart Association (AHA) defined a simplified 7-item tool to promote ideal cardiovascular health $(\mathrm{CVH})$, including both modifiable behaviours and objective measures, such as the following $\mathrm{CVH}$ metrics: physical activity, diet, body mass index (BMI), smoking, blood pressure, serum glucose and total serum cholesterol [3]. This approach emphasizes primordial prevention in which efforts are focused on preventing the development of risk factors, which is in contrast to primary or secondary prevention in which the focus is on the prevention of the first occurrence or recurrence of CVDs. Achieving a greater number of ideal $\mathrm{CVH}$ metrics has been reported to be associated with a lower risk of CVD incidence and mortality [4-7].

Few studies have investigated the joint effects of the 7 $\mathrm{CVH}$ metrics on the subclinical impairments of CVDs [8-10]. Most of these studies have investigated a single subclinical marker or a few makers (mainly aortic arch plaque, coronary artery calcification or common CIMT), and the studies have been relatively limited to US and European populations $[8,11]$. Accordingly, the aim of the present study was to simultaneously investigate both the biomarkers and markers of subclinical impairments of CVDs with ideal CVH metrics in a community-based cohort in a Chinese population.

\section{Methods}

\section{Study population}

A cross-sectional study was performed. We retrospectively analysed clinical data from 4686 participants (aged 20 years and older) who attended a routine health check-up in the
Health Management Centre of the Third Xiangya Hospital from July 2014 to August 2016. The population comprised white-collar workers (including teachers, professional technical personnel, civil servants, and administrative staff) living in Changsha, which is the capital city of Hunan province in central south China. The inclusion criteria were as follows: (1) no self-reported history of CVDs, including myocardial infarction or angina pectoris, heart failure, stroke or transient ischaemic attack; (2) no cardiomyopathy; (3) no malignancy; (4) no peripheral artery disease; (5) no chronic kidney disease; and (6) no data missing from the analysis. The study flowchart is presented in Fig. 1. Finally, 3009 subjects (2002 males, age range $24-85$ years) were enrolled. The study protocol was approved by the Ethics Review Committee of our hospital. All participants agreed to study participation and provided informed consent.

\section{Assessment of $\mathrm{CVH}$ metrics}

The $\mathrm{CVH}$ score was calculated according to the AHA guidelines by summing the $7 \mathrm{CVH}$ metrics as dichotomous variables, with 0 reflecting a poor and 1 an ideal $\mathrm{CVH}$ status [3]. We used alternative measures for the dietary and physical activity metrics in this study. Ideal health behaviours and factors were defined as follows: no history of smoking or quit smoking $>12$ months previously, moderate physical activity for $>150 \mathrm{~min}$ per week or vigorous physical activity for $>75$ min per week, 4 to 5 items of dietary status [with cereals and legumes as the basic food; $\geq 500 \mathrm{~g}$ (equivalent to 1 "Jin") of vegetables and fruits/day, $<100 \mathrm{~g}$ (equivalent to 2 "Liang") of

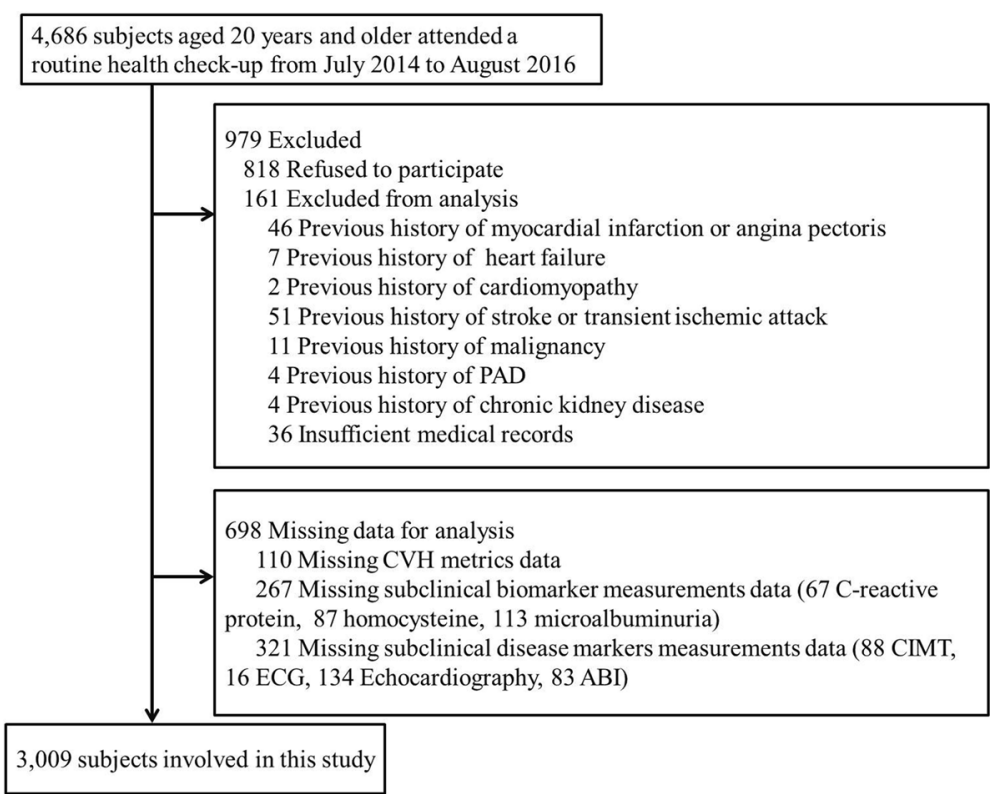

Fig. 1 Study flowchart. PAD, peripheral artery disease; CVH, cardiovascular health; CIMT, carotid intima-media thickness; ECG, electrocardiogram; $A B I$, Ankle-brachial index 
red meat/day, regular (in most weeks) intake of unprocessed fish and/or soybean products, and a preference for non-salty food] [12], BMI $<25 \mathrm{~kg} / \mathrm{m}^{2}$, untreated systolic BP $<120 \mathrm{mmHg}$ and diastolic BP $<80 \mathrm{mmHg}$, untreated fasting blood glucose level $<5.6 \mathrm{mmol} / \mathrm{L}(100 \mathrm{mg} / \mathrm{dL})$, and untreated total cholesterol level $<5.2 \mathrm{mmol} / \mathrm{L}(200 \mathrm{mg} / \mathrm{dL})$. Participants who had 0-2, 3-4 and 5-7 health metrics at the ideal level were defined as having poor, intermediate and ideal CVH statuses, respectively [3].

Information concerning smoking, physical activity, and dietary intake was collected by questionnaires administered by well-trained staff blinded to the clinical data. The resting systolic blood pressure (SBP), diastolic blood pressure (DBP), and BMI were measured using standard methods [13]. The biochemical variables, including total cholesterol (TC) and fasting plasma glucose (FPG), were measured using an autoanalyser (Olympus, AU400, Japan) in the central laboratory of our hospital.

\section{Subclinical biomarker measurements}

The three biomarkers represented the following distinct biological pathways: vascular inflammation state (CRP), vascular endothelial function and oxidative stress (homocysteine), and microvascular structural alteration (microalbuminuria). Blood samples were drawn by venepuncture after at least $10 \mathrm{~h}$ of overnight fasting. CRP was measured using a high-sensitivity nephelometry assay (Cias Latex CRP-H; Kanto Chemical, Tokyo, Japan). Homocysteine was measured using a chemiluminescent immunoassay (Centaur XP; Siemens, Deerfield, IL, USA). Urine was collected in the morning. Microalbuminuria was measured using kit reagents and the ProSpec nephelometric analyser (Dade Behring GMBH, Marburg, Germany).

\section{Subclinical disease marker measurements}

Five subclinical atherosclerosis phenotypes were measured for this study. B-mode ultrasonography examinations of the carotid arteries were performed by an experienced sonographer using the Siemens Acuson

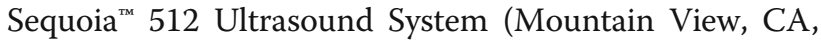
USA) with a $12-\mathrm{MHz}(9-14)$ linear matrix array transducer with the patient in the supine position. CIMT was measured bilaterally on 3 segments: the distal common carotid artery $(1 \mathrm{~cm}$ proximal to dilation of the carotid bulb), the carotid artery bifurcation, and the proximal internal carotid arteries (1-cm section of the internal carotid arteries immediately distal to the flow divider). The means of these segments were estimated for both the left and right sides. An increased CIMT was defined as $>900 \mu \mathrm{m}$, and a carotid plaque was defined as a focal structure that intruded into the arterial lumen by $\geq 0.5 \mathrm{~mm}$ or $50 \%$ of the value of the neighbouring carotid IMT or that measured $\geq 1.5 \mathrm{~mm}$ from the mediaadventitia boundary to the intima-lumen boundary [14].
The presence of LV hypertrophy was defined by electrocardiogram (ECG), echocardiography, or both methods. A standard 12-lead computerized resting ECG was obtained with the patient in the supine position. The Sokolow-Lyon index (SV1 + RV5/RV6, mm) was used to evaluate ECGLV hypertrophy $(>35 \mathrm{~mm})$ [15]. Echocardiography was performed using the GE Vivid 7 ultrasound scanner (GE Vingmed ultrasound) with a $2.5-\mathrm{MHz}$ linear transducer according to the American Society of Echocardiography recommendations [16]. The left ventricular mass (LVM) was calculated as follows: LV mass $(\mathrm{g})=0.8\{1.04$ [(LVEDD + PW + IVS) 3 - (LVEDD)3] +0.6 , in which LVEDD was the left ventricular end-diastolic diameter, and PW and IVS were the posterior wall thickness and the interventricular septum thickness at end diastole from an average of $\geq 3$ cardiac cycles, respectively. Then, the LV mass was normalized for the body surface area (the DuBois formula: $0.007184 \times$ height $(\mathrm{cm}) 0.725 \times$ weight $(\mathrm{kg}) 0.425)$ [17] to obtain the LVM index (LVMI). LV hypertrophy echocardiography was defined as an LVMI $>116 \mathrm{~g} / \mathrm{m} 2$ for men and $>95 \mathrm{~g} / \mathrm{m} 2$ for women [18].

The LV ejection fraction was estimated based on a visual assessment of the left ventricular contractile performance and wall motion in multiple two-dimensional views. LV fractional shortening was calculated as (LVEDD - LVESD)/LVEDD, in which LVESD was the LV end-systolic diameter. LV systolic dysfunction was defined as LV fractional shortening $<0.29$ by M-mode, evidence of an ejection fraction $<50 \%$ using the twodimensional method, or both criteria [19].

The ankle-brachial index (ABI) was measured with an automatic waveform analyser (Colin Co., Komaki, Japan). Briefly, after taking at least a 5-min rest in the supine position, the subjects attached cuffs around their upper arms and ankles. The ABI was calculated as the ratio of the average SBP at the ankle of each leg divided by the average SBP in the arm. An $\mathrm{ABI} \leq 0.9$ was considered ABI-defined peripheral artery disease (PAD) [20].

\section{Statistical analysis}

Continuous data were expressed as means \pm standard deviations (SDs) and median with 25th and 75th percentiles. The data were compared using Student's t-test for normally distributed variables and the Mann-Whitney $\mathrm{U}$ test for non-normally distributed variables. Categorical variables were expressed as percentages and were compared using the chi-square test.

We logarithmically transformed all subclinical biomarkers to normalize their skewed distributions. Subclinical atherosclerosis was defined as the presence of at least one component of the five subclinical disease markers: increased CIMT, presence of a carotid plaque, LV hypertrophy (by ECG or echocardiography), LV systolic dysfunction (by echocardiography), or reduced ABI. 
Then, we constructed a subclinical marker score ranging from 0 to 5 to calculate the number of the five abnormal measures. First, a linear regression model was used to evaluate the association between the $\mathrm{CVH}$ score (a continuous variable) and each subclinical biomarker and between the $\mathrm{CVH}$ score and each subclinical disease marker simultaneously stratified by gender. Standard regression beta $(\beta)$ coefficients were calculated. We fitted 3 multivariate models. Model 1 had no adjustments. Model 2 adjusted for age and gender. Model 3 additionally adjusted for the education level. Next, we used a logistic regression model to assess the association between the numbers of subclinical disease marker components (subclinical marker score $\geq 1$ vs. $0, \geq 2$ vs. 0 or 1 , and $\geq 3$ vs. 0,1 , or 2 ) and the $\mathrm{CVH}$ score (a continuous variable). The odds ratios (ORs) and 95\% CIs were calculated. The adjusted models were the same as described above.

To examine the robustness of our results, sensitivity analyses were performed using a 14-point $\mathrm{CVH}$ score, which included 7 components based on the following categories: $0=$ poor, $1=$ intermediate, and $2=$ ideal (Additional file 1). All statistical analyses were performed using the SPSS software version 20.0 (IBM). P values less than 0.05 were considered significant.

\section{Results}

\section{General characteristics of the participants}

A total of 3009 individuals were enrolled in this crosssectional study. The mean age was $48.6 \pm 9.7$ years, and $33.5 \%$ of the participants were female. Table 1 shows the characteristics of the participants stratified by gender. The females tended to be older and had lower levels of education. The parameters, including the BMI, SBP, DBP, and FPG, and the proportions of participants who were current drinkers, had diabetes mellitus, and used diabetes medication were significantly higher in the males than in the females. Conversely, the high-density lipoprotein (HDL) levels were significantly higher in the females than in the males. The TC and low-density lipoprotein (LDL) values, the proportion of patients with hypertension, and the use of antihypertensive and lipidlowering medications were not significantly different between the males and females.

The mean $\pm \mathrm{SD}$ of the $\mathrm{CVH}$ score was $2.8 \pm 1.3$. Only $0.2 \%$ of the study participants had the 7 ideal $\mathrm{CVH}$ metrics $(\mathrm{CVH}$ score $=7)$, accounting for $0 \%$ of the males and $0.5 \%$ of the females. The CVH score distribution differed between the females and males. The males most frequently had $\mathrm{CVH}$ scores of 0 to 3 , whereas the women most commonly had $\mathrm{CVH}$ scores of 4 to 7 (Fig. 2). The levels of the three subclinical biomarkers (homocysteine, CRP, and microalbuminuria) were all higher in the males than those in the females (all $P<0.01)$. This trend was consistent with the subclinical disease markers. The males had significantly greater percentages of increased CIMT, LV hypertrophy, and LV systolic dysfunction than the females. Conversely, carotid plaques did not differ by gender, and reduced $A B I$ occurred less frequently in the males.

\section{Distribution of the subclinical biomarkers/subclinical disease markers by CVH category}

The characteristics of the subclinical biomarkers/subclinical disease markers by poor, intermediate and ideal $\mathrm{CVH}$ statuses are reported in Table 2. When the CVH score increased from poor to ideal, the subclinical biomarker levels and the proportion of subclinical disease markers decreased significantly (all $P<0.05$ ).

\section{Association between the subclinical biomarkers and the CVH score}

Table 3 shows the associations between each logtransformed subclinical biomarker level and the $\mathrm{CVH}$ score. We found that the homocysteine, CRP, and microalbuminuria concentrations were significantly inversely related to the $\mathrm{CVH}$ score $(P<0.001$ for all). The associations remained robust after adjusting for gender, age, and education level. The stratified analyses found that this significant negative correlation was evident in both females and males $(P<0.001$ for all).

\section{Association between the subclinical disease markers and the $\mathrm{CVH}$ score}

An increased CIMT was present in 422 participants (14.0\%), carotid plaque was present in 805 (26.8\%), LV hypertrophy was present in 132 (15.1\%), LV systolic dysfunction was present in $63(2.1 \%)$, and peripheral arterial disease was present in 35 (1.2\%). The mean \pm SD of the subclinical disease marker score was $0.59 \pm 0.76$. Table 4 demonstrates a significant association between the $\mathrm{CVH}$ score (continuous variable) and each subclinical disease marker $(P<0.05$ for all). Table 5 shows the association between the $\mathrm{CVH}$ score and the prevalence of the subclinical disease markers (categorical variable $\geq 1$ vs. $0, \geq 2$ vs. 0 or 1 , and $\geq 3$ vs. 0,1 , or 2 ). We found that increasing $\mathrm{CVH}$ scores indicated a pattern of decreasing odds of the prevalence of subclinical disease markers $(P<0.001$ for all). This negative correlation remained significant in the fully adjusted models and in the female and male subgroups, particularly in the females $(P<0.05$ for all $)$.

\section{Sensitivity analyses}

An additional 14-point ideal CVH score was created. The analyses were repeated for both the subclinical biomarkers and the subclinical disease markers. The results were in accordance with the 7-point ideal $\mathrm{CVH}$ scores (Additional files 2, 3 and 4). 
Table 1 Clinical characteristics of the study sample

\begin{tabular}{|c|c|c|c|c|}
\hline Characteristics & $\begin{array}{l}\text { Overall } \\
(n=3009)\end{array}$ & $\begin{array}{l}\text { Female } \\
(n=1007)\end{array}$ & $\begin{array}{l}\text { Male } \\
(n=2002)\end{array}$ & $P$ Value \\
\hline Age, y & $48.6 \pm 9.7$ & $49.9 \pm 9.9$ & $48.0 \pm 9.6$ & $<0.001$ \\
\hline \multicolumn{5}{|l|}{ Education, n (\%) } \\
\hline Elementary school & $206(6.9)$ & 135(13.4) & $71(3.6)$ & \multirow[t]{3}{*}{$<0.001$} \\
\hline High school & $1353(45.0)$ & $538(53.4)$ & $815(40.7)$ & \\
\hline College or above & $1450(48.2)$ & $334(33.2)$ & $1116(55.7)$ & \\
\hline Body mass index, $\mathrm{kg} / \mathrm{m}^{2}$ & $24.9 \pm 3.3$ & $23.5 \pm 3.1$ & $25.5 \pm 3.2$ & $<0.001$ \\
\hline Systolic blood pressure, $\mathrm{mmHg}$ & $128.3 \pm 16.6$ & $125.5 \pm 18.5$ & $129.7 \pm 15.3$ & $<0.001$ \\
\hline Diastolic blood pressure, $\mathrm{mmHg}$ & $81.3 \pm 11.3$ & $76.3 \pm 11.0$ & $83.6 \pm 10.8$ & $<0.001$ \\
\hline Hypertension, n (\%) & 409 (13.6) & $125(12.4)$ & $284(14.2)$ & 0.195 \\
\hline Antihypertensive medication, $\mathrm{n}(\%)$ & $373(12.4)$ & $109(10.8)$ & $264(13.2)$ & 0.069 \\
\hline Fasting glucose, $\mathrm{mmol} / \mathrm{L}$ & $5.4 \pm 1.4$ & $5.2 \pm 1.0$ & $5.5 \pm 1.6$ & $<0.001$ \\
\hline Diabetes mellitus, $n(\%)$ & $134(4.5)$ & $22(2.2)$ & $112(5.6)$ & $<0.001$ \\
\hline Diabetes medication, $n$ (\%) & $111(3.7)$ & $21(2.1)$ & $90(4.5)$ & $<0.001$ \\
\hline Total cholesterol, mmol/L & $5.2 \pm 1.0$ & $5.2 \pm 1.0$ & $5.2 \pm 1.0$ & 0.199 \\
\hline HDL-cholesterol, mmol/L & $1.4 \pm 0.4$ & $1.6 \pm 0.4$ & $1.3 \pm 0.3$ & $<0.001$ \\
\hline LDL-cholesterol, mmol/L & $2.9 \pm 0.9$ & $2.9 \pm 0.9$ & $2.9 \pm 0.9$ & 0.95 \\
\hline Lipid-lowering medication, n (\%) & $47(1.6)$ & $10(1.0)$ & $37(1.8)$ & 0.086 \\
\hline \multicolumn{5}{|l|}{ Cardiovascular health metrics } \\
\hline Ideal diet, $n(\%)$ & $102(3.4)$ & $51(5.1)$ & $51(2.5)$ & 0.001 \\
\hline Ideal smoking status, $n(\%)$ & $1944(64.6)$ & $978(97.1)$ & $966(48.3)$ & $<0.001$ \\
\hline Ideal physical activity, n (\%) & $339(11.3)$ & $112(11.1)$ & $227(11.3)$ & 0.859 \\
\hline Ideal BMI, $n(\%)$ & $1591(52.9)$ & $726(72.1)$ & $865(43.2)$ & $<0.001$ \\
\hline Ideal Blood pressure, $n(\%)$ & $797(26.5)$ & $391(38.8)$ & $406(20.3)$ & $<0.001$ \\
\hline Ideal Total cholesterol, n (\%) & $1472(48.9)$ & $517(51.3)$ & $955(47.7)$ & 0.060 \\
\hline Ideal Fasting glucose, $n$ (\%) & $2258(75.0)$ & $826(82.0)$ & $1432(71.5)$ & $<0.001$ \\
\hline Ideal CVH score & $2.8 \pm 1.3$ & $3.6 \pm 1.2$ & $2.5 \pm 1.2$ & $<0.001$ \\
\hline \multicolumn{5}{|l|}{ Subclinical biomarkers, median (Q1, Q3) } \\
\hline Homocysteine, $\mu \mathrm{mol} / \mathrm{L}$ & $9.4(7.6,11.6)$ & $7.8(6.3,9.3)$ & $10.4(8.6,12.5)$ & $<0.001$ \\
\hline C-reactive protein, mg/L & $1.5(0.8,2.6)$ & $1.3(0.7,2.4)$ & $1.6(0.8,2.7)$ & $<0.001$ \\
\hline Microalbuminuria, mg/L & $9.5(5.3,16.8)$ & $7.0(4.0,13.0)$ & $11.0(6.0,18.0)$ & 0.005 \\
\hline \multicolumn{5}{|l|}{ Subclinical disease markers } \\
\hline 1. Increased CIMT, n (\%) & $422(14.0)$ & $118(11.7)$ & $30(15.2)$ & 0.010 \\
\hline 2. Presence of carotid plaque, $n(\%)$ & $805(26.8)$ & $250(24.8)$ & $555(27.7)$ & 0.097 \\
\hline \multicolumn{5}{|l|}{ 3. LV hypertrophy by ECG/Echocardiography } \\
\hline LV hypertrophy by ECG (Sokolow-Lyon criteria), $n$ (\%) & $132(4.4)$ & $18(1.8)$ & $114(5.7)$ & $<0.001$ \\
\hline LV mass-to-BSA ratio, g/m2 & $92.4 \pm 18.2$ & $77.8 \pm 14.2$ & $99.7 \pm 15.3$ & $<0.001$ \\
\hline LV hypertrophy by Echocardiography, n (\%) & $341(11.3)$ & $84(8.3)$ & $257(12.8)$ & $<0.001$ \\
\hline $\begin{array}{l}\text { LV hypertrophy by ECG or Echocardiography, } \\
n(\%)\end{array}$ & $453(15.1)$ & $100(1.0)$ & $353(17.6)$ & $<0.001$ \\
\hline \multicolumn{5}{|l|}{ 4. LV systolic dysfunction by echocardiography } \\
\hline Fractional shortening & $0.37 \pm 0.04$ & $0.38 \pm 0.04$ & $0.37 \pm 0.04$ & $<0.001$ \\
\hline LV systolic dysfunction, $n$ (\%) & $63(2.1)$ & $11(1.1)$ & $52(2.6)$ & $<0.001$ \\
\hline \multicolumn{5}{|l|}{ 5. Peripheral arterial disease by $A B I$} \\
\hline $\mathrm{ABI} \leq 0.9, n(\%)$ & $35(1.2)$ & $19(1.9)$ & $16(0.8)$ & 0.011 \\
\hline
\end{tabular}


Table 1 Clinical characteristics of the study sample (Continued)

\begin{tabular}{llll}
\hline Characteristics & $\begin{array}{l}\text { Overall } \\
(n=3009)\end{array}$ & $\begin{array}{l}\text { Female } \\
(n=1007)\end{array}$ & $\begin{array}{l}\text { Male } \\
(n=2002)\end{array}$ \\
\hline Composite of subclinical disease markers & & & $P$ Value \\
At least $1, n(\%)$ & $1339(44.5)$ & $377(37.4)$ & $962(48.1)$ \\
At least 2, n (\%) & $383(12.7)$ & $105(10.4)$ & $278(13.9)$ \\
$\geq 3, \mathrm{n}(\%)$ & $56(1.9)$ & $16(1.6)$ & $40(2.0)$ \\
Mean score & $0.59 \pm 0.76$ & $0.49 \pm 0.72$ & $0.64 \pm 0.77$
\end{tabular}

Q1, quartile 1; Q3, quartile 3; $L D L$, low-density lipoprotein; $H D L$, high-density lipoprotein; $C V H$, cardiovascular health; CIMT, carotid intima-media thickness; $L V$, left ventricular; $B S A$, body surface area; $E C G$, electrocardiogram; $A B l$, ankle-brachial index

\section{Discussion}

In this study, we investigated the relationships between subclinical biomarkers and subclinical disease markers of CVDs with ideal $\mathrm{CVH}$ metrics in a general urban population from central south China. We found that the $\mathrm{CVH}$ score was inversely related to both the biomarker levels (homocysteine, CRP, and microalbuminuria) and the presence of subclinical disease markers (increased CIMT, carotid plaques, LV hypertrophy, LV systolic dysfunction, and peripheral arterial disease). A similar negative correlation was observed in the female and male subgroups, and consistent trends were observed with the 14-point $\mathrm{CVH}$ score. To the best of our knowledge, this study is the first to correlate the relatively comprehensive subclinical profile of CVDs with ideal CVH in Chinese adults.

The biomarkers used in the study represent different biological pathways of atherosclerosis. CRP is the most clinically accepted inflammatory biomarker used to predict future cardiovascular events in asymptomatic individuals [21]. Hyperhomocysteinaemia has multiple potentially toxic effects on atherogenesis, including damaging endothelial cells, reducing the flexibility of vessels, and adversely affecting the process of homeostasis [22, 23]. Microalbuminuria is predictive of worsening microvascular disease in the kidney, reflects a generalized abnormality of vascular function, and is associated with a strong risk of increasing CVDs [24]. Notably, the items

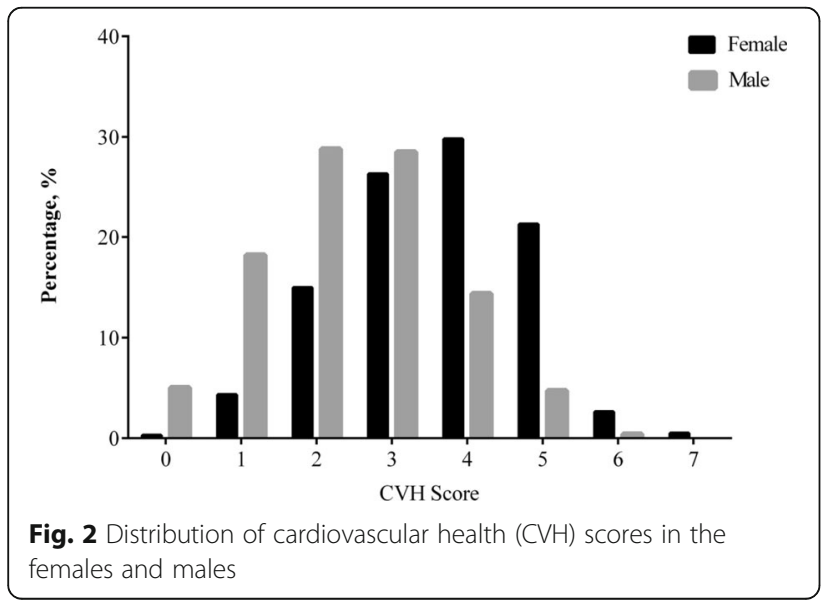

in the $\mathrm{CVH}$ metrics are risk factors associated with the biomarkers. BMI, smoking, and diabetes are associated with CRP [25]. Smoking, lack of exercise, and hypertension are related to hyperhomocysteinaemia [26]. Diabetes, hypertension, smoking, blood lipids, BMI, and dietary protein are correlated with microalbuminuria [27]. The observed associations of the selected biomarkers with the $\mathrm{CVH}$ scores suggest that a range of biological pathways can mediate the favourable effects of the $\mathrm{CVH}$ score on subclinical atherosclerosis or disease.

The subclinical stage serves as an intermediate endpoint for the risk evaluation and optimal treatment. We used previously validated markers of subclinical disease. An increased CIMT, carotid plaques and reduced ABI were indicators for macrovascular disease (coronary heart disease, myocardial infarction, PAD and stroke), whereas LV hypertrophy and LV systolic dysfunction were indicators of target organ damage. Among the composite of the subclinical disease markers, the majority of the participants (55.5\%) had no subclinical disease markers, only $12.7 \%$ had at least 2 components, and $1.9 \%$ had $\geq 3$ components, which was consistent with a previous study [9]. There may be several reasons for this status. First, the median age in our cohort was relatively young (48.6 years) with regard to CVD risk. Second, the prevalence of hypertension and diabetes was low, implying a lower CVD risk. Third, many CVH metrics (e.g., mean lipid, fasting glucose, and BP values) were inside the normal ranges, suggesting that the subjects were either healthy or well treated.

Our study found that subclinical impairments were independently associated with low $\mathrm{CVH}$ scores. Low $\mathrm{CVH}$ scores may primarily contribute to chronic subclinical damage, leading to CVDs via subclinical impairments. Previous studies have largely focused on the association of subclinical stages with each individual behaviour or health factor [28]. However, many of these factors coexist or occur in aggregation and thus may be interwoven into multiple biologically related pathways. The association in the present study reflected the combined and cumulative effects of exposure to risk factors on subclinical lesions and thus provided more accurate information compared to exploring each factor individually. 
Table 2 Characteristics of subclinical impairments according to the CVH score categories

\begin{tabular}{|c|c|c|c|c|}
\hline Characteristics & $\begin{array}{l}\text { Poor CVH } \\
(0-2)\end{array}$ & $\begin{array}{l}\text { Intermediate CVH } \\
(3-4)\end{array}$ & $\begin{array}{l}\text { Ideal CVH } \\
(5-7)\end{array}$ & $P$ Value \\
\hline Number, $n$ & 1238 & 1423 & 348 & \\
\hline \multicolumn{5}{|l|}{ Subclinical biomarkers, median (Q1, Q3) } \\
\hline Homocysteine, $\mu \mathrm{mol} / \mathrm{L}$ & $10.2(8.3,12.3)$ & $9.0(7.3,11.0)$ & $7.5(6.2,9.2)$ & $<0.001$ \\
\hline C-reactive protein, mg/L & $1.9(1.1,3.2)$ & $1.3(0.7,2.3)$ & $0.9(0.58,1.6)$ & $<0.001$ \\
\hline Microalbuminuria, mg/L & $11.6(6.1,21.6)$ & $8.6(4.9,14.3)$ & $5.2(2.0,9.8)$ & $<0.001$ \\
\hline \multicolumn{5}{|l|}{ Subclinical disease markers, $n(\%)$} \\
\hline 1. Increased CIMT & $214(17.3)$ & $184(12.9)$ & $24(6.9)$ & $<0.001$ \\
\hline 2. Presence of carotid plaque & $396(32.0)$ & $364(25.6)$ & $45(12.9)$ & $<0.001$ \\
\hline 3. LV hypertrophy by ECG/echocardiography & $215(17.4)$ & $211(14.8)$ & $27(7.8)$ & $<0.001$ \\
\hline 4. LV systolic dysfunction by echocardiography & $39(3.2)$ & $23(1.6)$ & $1(0.3)$ & 0.001 \\
\hline 5. Peripheral arterial disease by $\mathrm{ABI} \leq 0.9$ & $22(1.8)$ & $13(0.9)$ & 0 & 0.012 \\
\hline \multicolumn{5}{|l|}{ Composite of subclinical disease markers } \\
\hline At least $1, n(\%)$ & $643(51.9)$ & $605(42.5)$ & $91(26.3)$ & $<0.001$ \\
\hline At least $2, n(\%)$ & $201(16.27)$ & $167(11.7)$ & $15(4.3)$ & $<0.001$ \\
\hline$\geq 3, n(\%)$ & $31(2.5)$ & $24(1.7)$ & $1(0.3)$ & 0.021 \\
\hline Mean score & $0.71 \pm 0.80$ & $0.56 \pm 0.74$ & $0.31 \pm 0.56$ & $<0.001$ \\
\hline
\end{tabular}

Q1, quartile 1; Q3, quartile 3; $C V H$, cardiovascular health; CIMT, carotid intima-media thickness; $L V$, left ventricular; $B S A$, body surface area; $E C G$, electrocardiogram; $A B I$, ankle-brachial index

Previous studies also uncovered associations between $\mathrm{CVH}$ and subclinical impairments. In the Paris Prospective Study III, more ideal CVH metrics were positively associated with a lower presence of subclinical disease markers of carotid structure and function (common CIMT, carotid plaques, lower Young's elastic modulus, and carotid dispensability coefficient) among community subjects aged 50 to 75 years [10]. In the Young Finns Study, Laitinen et al. reported that a greater number of average lifetime $\mathrm{CVH}$ metrics and favourable changes in the $\mathrm{CVH}$ status in childhood or young adulthood were associated with a reduced risk of coronary artery calcification, carotid dispensability, and increased CIMT in middle age [8]. In the Framingham study, higher ideal $\mathrm{CVH}$ scores were inversely related to a composite measure of subclinical CVDs (including higher carotid artery IMT or stenosis, LV systolic dysfunction, LV hypertrophy, microalbuminuria, or reduced ABI) [9].

Table 3 Associations between the CVH score (per 1-unit increase) and the log-transformed subclinical biomarker level stratified by gender

\begin{tabular}{|c|c|c|c|c|c|c|}
\hline & \multicolumn{2}{|l|}{ Overall } & \multicolumn{2}{|l|}{ Female } & \multicolumn{2}{|l|}{ Male } \\
\hline & $\beta$ Coefficient $(95 \% \mathrm{Cl})$ & $P$ Value & $\beta$ Coefficient (95\% Cl) & $P$ Value & $\beta$ Coefficient $(95 \% \mathrm{Cl})$ & $P$ Value \\
\hline \multicolumn{7}{|c|}{ Homocysteine } \\
\hline Model 1 & $-0.228(-1.926$ to -1.415$)$ & $<0.001$ & $-0.188(-1.827$ to -0.935$)$ & $<0.001$ & $-0.087(-0.909$ to -0.299$)$ & $<0.001$ \\
\hline Model 2 & $-0.097(-0.960$ to -0.456$)$ & $<0.001$ & $-0.115(-1.271$ to -0.412$)$ & $<0.001$ & $-0.084(-0.890$ to -0.277$)$ & $<0.001$ \\
\hline Model 3 & $-0.092(-0.930$ to -0.426$)$ & $<0.001$ & $-0.111(-1.242$ to -0.388$)$ & $<0.001$ & $-0.081(-0.873$ to -0.259$)$ & $<0.001$ \\
\hline \multicolumn{7}{|c|}{ C-reactive protein } \\
\hline Model 1 & $-0.233(-0.815$ to -0.603$)$ & $<0.001$ & $-0.316(-1.032$ to -0.709$)$ & $<0.001$ & $-0.177(-0.621$ to -0.378$)$ & $<0.001$ \\
\hline Model 2 & $-0.190(-0.674$ to -0.479$)$ & $<0.001$ & $-0.231(-0.795$ to -0.474$)$ & $<0.001$ & $-0.175(-0.617$ to -0.373$)$ & $<0.001$ \\
\hline Model 3 & $-0.182(-0.652$ to -0.457$)$ & $<0.001$ & $-0.219(-0.764$ to -0.441$)$ & $<0.001$ & $-0.172(-0.605$ to -0.363$)$ & $<0.001$ \\
\hline \multicolumn{7}{|c|}{ Microalbuminuria } \\
\hline Model 1 & $-0.296(-0.929$ to -0.729$)$ & $<0.001$ & $-0.192(-0.587$ to -0.305$)$ & $<0.001$ & $-0.244(-0.677$ to -0.476$)$ & $<0.001$ \\
\hline Model 2 & $-0.228(-0.734$ to -0.546$)$ & $<0.001$ & $-0.167(-0.521$ to -0.257$)$ & $<0.001$ & $-0.245(-0.676$ to -0.475$)$ & $<0.001$ \\
\hline Model 3 & $-0.224(-0.720$ to -0.534$)$ & $<0.001$ & $-0.163(-0.511$ to -0.247$)$ & $<0.001$ & $-0.238(-0.662$ to -0.463$)$ & $<0.001$ \\
\hline
\end{tabular}

Values are standard regression coefficient betas $(95 \% \mathrm{Cl})$ and $\mathrm{p}$ values. Each beta coefficient represents the change in log-biomarker per 1 -unit increase in the CVH score. Model 1, unadjusted; model 2, adjusted for age and sex; model 3, adjusted for age, sex and level of education 
Table 4 Associations between the CVH score (per 1- unit increase) and each component of the subclinical disease markers stratified by gender

\begin{tabular}{|c|c|c|c|c|c|c|}
\hline & \multicolumn{2}{|l|}{ Overall } & \multicolumn{2}{|l|}{ Female } & \multicolumn{2}{|l|}{ Male } \\
\hline & $\beta$ Coefficient $(95 \% \mathrm{Cl})$ & $P$ Value & $\beta$ Coefficient $(95 \% \mathrm{Cl})$ & $P$ Value & $\beta$ Coefficient $(95 \% \mathrm{Cl})$ & $P$ Value \\
\hline \multicolumn{7}{|c|}{ Increased CIMT } \\
\hline Model 1 & $-0.115(-0.580$ to -0.305$)$ & $<0.001$ & $-0.170(-0.873$ to -0.412$)$ & $<0.001$ & $-0.076(-0.412$ to -0.110$)$ & 0.001 \\
\hline Model 2 & $-0.060(-0.363$ to -0.100$)$ & $<0.001$ & $-0.087(-0.551$ to -0.104$)$ & 0.004 & $-0.071(-0.407$ to -0.085$)$ & 0.003 \\
\hline Model 3 & $-0.060(-0.362$ to -0.099$)$ & $<0.001$ & $-0.081(-0.527$ to -0.082$)$ & 0.007 & $-0.072(-0.527$ to -0.082$)$ & 0.002 \\
\hline \multicolumn{7}{|c|}{ Presence of carotid plaque } \\
\hline Model 1 & $-0.127(-0.494$ to -0.279$)$ & $<0.001$ & $-0.261(-0.902$ to -0.566$)$ & $<0.001$ & $-0.061(-0.291$ to -0.048$)$ & 0.006 \\
\hline Model 2 & $-0.071(-0.324$ to -0.108$)$ & $<0.001$ & $-0.114(-0.504$ to -0.139$)$ & 0.001 & $-0.055(-0.284$ to -0.021$)$ & 0.023 \\
\hline Model 3 & $-0.069(-0.315$ to -0.100$)$ & $<0.001$ & $-0.114(-0.503$ to -0.140$)$ & 0.001 & $-0.053(-0.278$ to -0.015$)$ & 0.029 \\
\hline \multicolumn{7}{|c|}{ LV hypertrophy by ECG/echocardiography } \\
\hline Model 1 & $-0.118(-0.648$ to -0.348$)$ & $<0.001$ & $-0.193(-1.116$ to -0.582$)$ & $<0.001$ & $-0.062(-0.391$ to -0.067$)$ & 0.006 \\
\hline Model 2 & $-0.073(-0.448$ to -0.171$)$ & $<0.001$ & $-0.141(-0.872$ to -0.366$)$ & $<0.001$ & $-0.058(-0.377$ to -0.049$)$ & 0.011 \\
\hline Model 3 & $-0.066(-0.419$ to -0.141$)$ & $<0.001$ & $-0.130(-0.825$ to -0.318$)$ & $<0.001$ & $-0.053(-0.363$ to -0.033$)$ & 0.018 \\
\hline \multicolumn{7}{|c|}{ LV systolic dysfunction by echocardiography } \\
\hline Model 1 & $-0.083(-1.113$ to -0.445$)$ & $<0.001$ & $-0.105(-1.945$ to -0.506$)$ & 0.001 & $-0.059(-0.802$ to -0.119$)$ & 0.008 \\
\hline Model 2 & $-0.056(-0.826$ to -0.217$)$ & 0.001 & $-0.060(-1.381$ to -0.027$)$ & 0.042 & $-0.058(-0.791$ to -0.108$)$ & 0.010 \\
\hline Model 3 & $-0.053(-0.803$ to -0.195$)$ & 0.001 & $-0.059(-1.358$ to -0.012$)$ & 0.046 & $-0.056(-0.778$ to -0.096$)$ & 0.012 \\
\hline \multicolumn{7}{|c|}{ Peripheral arterial disease by $\mathrm{ABI} \leq 0.9$} \\
\hline Model 1 & $-0.053(-1.109$ to -0.215$)$ & 0.004 & $-0.108(-1.512$ to -0.413$)$ & 0.001 & $-0.060(-1.440$ to -0.221$)$ & 0.008 \\
\hline Model 2 & $-0.065(-1.218$ to -0.406$)$ & $<0.001$ & $-0.087(-1.291$ to -0.265$)$ & 0.003 & $-0.058(-1.415$ to -0.195$)$ & 0.010 \\
\hline Model 3 & $-0.062(-1.182$ to -0.317$)$ & $<0.001$ & $-0.081(-1.236$ to -0.214$)$ & 0.005 & $-0.057(-1.401$ to -0.181$)$ & 0.011 \\
\hline
\end{tabular}

Values are standard regression coefficient betas $(95 \% \mathrm{Cl})$ and $p$ values. Each beta coefficient represents the change in log-biomarker per 1-unit increase in the CVH score. Model 1, unadjusted; model 2, adjusted for age and sex; model 3, adjusted for age, sex and level of education

Table 5 Associations between the CVH score (per 1- unit increase) and number of the subclinical disease markers stratified by gender

\begin{tabular}{|c|c|c|c|c|c|c|}
\hline & \multicolumn{2}{|l|}{ Overall } & \multicolumn{2}{|l|}{ Female } & \multicolumn{2}{|l|}{ Male } \\
\hline & OR $(95 \% \mathrm{Cl})$ & $P$ Value & OR $(95 \% \mathrm{Cl})$ & $P$ Value & OR $(95 \% \mathrm{Cl})$ & $P$ Value \\
\hline \multicolumn{7}{|l|}{$\geq 1$ vs. 0} \\
\hline Model 1 & $0.745(0.704-0.788)$ & $<0.001$ & $0.533(0.471-0.602)$ & $<0.001$ & $0.856(0.797-0.902)$ & $<0.001$ \\
\hline Model 2 & $0.802(0.749-0.858)$ & $<0.001$ & $0.676(0.591-0.774)$ & $<0.001$ & $0.857(0.791-0.928)$ & $<0.001$ \\
\hline Model 3 & $0.808(0.755-0.865)$ & $<0.001$ & $0.682(0.596-0.781)$ & $<0.001$ & $0.862(0.796-0.933)$ & $<0.001$ \\
\hline \multicolumn{7}{|c|}{$\geq 2$ vs. 0 or 1} \\
\hline Model 1 & $0.703(0.644-0.767)$ & $<0.001$ & $0.509(0.423-0.613)$ & $<0.001$ & $0.742(0.657-0.838)$ & $<0.001$ \\
\hline Model 2 & $0.724(0.654-0.802)$ & $<0.001$ & $0.627(0.513-0.766)$ & $<0.001$ & $0.744(0.658-0.840)$ & $<0.001$ \\
\hline Model 3 & $0.729(0.658-0.807)$ & $<0.001$ & $0.640(0.523-0.782)$ & 0.001 & $0.781(0.700-0.872)$ & $<0.001$ \\
\hline \multicolumn{7}{|c|}{$\geq 3$ vs. 0,1 , or 2} \\
\hline Model 1 & $0.594(0.463-0.761)$ & $<0.001$ & $0.465(0.298-0.698)$ & $<0.001$ & $0.573(0.422-0.778)$ & $<0.001$ \\
\hline Model 2 & $0.595(0.464-0.763)$ & $<0.001$ & $0.568(0.358-0.901)$ & 0.016 & $0.579(0.274-0.784)$ & $<0.001$ \\
\hline Model 3 & $0.603(0.484-0.752)$ & $<0.001$ & $0.583(0.368-0.925)$ & 0.022 & $0.623(0.468-0.830)$ & 0.001 \\
\hline
\end{tabular}

Values represent odds ratios (ORs) (95\% confidence interval [CI]) per 1-unit increase in cardiovascular health (CVH) score; $\geq 1$ denotes the presence of at least 1 component of increased carotid intima-media thickness, presence of carotid plaque, left ventricular hypertrophy (by ECG or echocardiography), left ventricular systolic dysfunction, or a reduced ankle-brachial index

Model 1, unadjusted; model 2, adjusted for age and sex; model 3, adjusted for age, sex and level of education 
The Special Turku Coronary Risk Factor Intervention Project for Children [STRIP] study reported that healthy $\mathrm{CVH}$ scores were directly associated with a lower aortic intima-media thickness and greater elasticity in adolescents [29]. Another study indicated that improvements in ideal cardiovascular health through midlife to late life were associated with a lower CVD prevalence and better cardiac structure and vascular functions, including carotid-femoral and brachial-ankle PWV (baPWV), in the elderly [30]. Similar results were obtained by Yan et al. in a Chinese population after assessing the association between the CVH score and baPWV [31]. Our study findings have confirmed and extended these results in Asian populations.

A global ideal $\mathrm{CVH}$ was rare $(0.2 \%)$ in our study, which was consistent with other studies with more representative samples (values of approximately 0.1 to $0.2 \%$ were reported in American and Chinese urban populations) [32-34]. Females had better $\mathrm{CVH}$ profiles and a lower prevalence of most subclinical disease markers than males, and the relationship between the $\mathrm{CVH}$ score and markers tended to be stronger among females than that among males, which was also in line with prior reports [35]. The exact mechanism underlying this observed gender difference warrants further investigation. Generally, females may be less exposed to cardiovascular risk factors, such as smoking, alcohol use and overweight, and they may receive more protective effects from endogenous oestrogens prior to reaching the premenopausal age $[36,37]$. Considering the above findings, we should attach great concerns to the primary care of CVDs, especially for males. In our study, women were more likely to be classified with a reduced ABI, which was consistent with previous epidemiological evidence showing that women had lower ABI values than men $[38,39]$. Gender differences in the ABI value may occur because men on average are taller than women, and men have a greater tendency to experience medial arterial calcinosis, which may avoid an ABI decline [40]. Although the gender differences in PAD must be explored, the intrinsic differences in the ABI criterion and measurements may confound the results [41].

The major strengths of this study include the large population-based sample, the use of a wide range of biomarkers and markers of subclinical data, and the performance of sensitivity analyses. However, some limitations to the present study should also be acknowledged. First, the cross-sectional design of our study only allowed the documentation of one-time sampling data that did not take the time-associated changes in the $\mathrm{CVH}$ scores into consideration, which might have improved the accuracy of our findings. Similarly, we did not explore the association between the $\mathrm{CVH}$ score and CVD incidence through prospective analyses. Second, some potential biomarkers, such as aldosterone, B-type natriuretic peptide (BNP), and growth differentiation factor-15 (GDF-15), and markers such as coronary artery calcium and lipoprotein-associated phospholipase A2 (Lp-PLA2) were not involved in these observed associations. Third, socioeconomic factors, such as family incomes, paying for medical expenses, frequency of health care visits, and the duration of treatment (antihypertensive, lipid-lowering diabetes and medication), might contribute to the relationship between $\mathrm{CVH}$ metrics and subclinical impairments, but we did not have this information. Finally, we used modified definitions of physical activity and diet that varied from the exact AHA definition to compute the scores for the $\mathrm{CVH}$ metrics.

\section{Conclusion}

In summary, the prevalence of ideal CVH was low in the adult population in central south China. The $\mathrm{CVH}$ score was associated with a favourable profile of subclinical biomarkers or markers of CVDs, thereby supporting the use of the $\mathrm{CVH}$ score as a reasonable and useful tool for assessing and preventing subclinical impairments, thus reducing the burden of CVDs. These findings may deepen our understanding of the biological mechanisms and processes underlying $\mathrm{CVH}$ and CVDs.

\section{Additional files}

\section{Additional file 1: Table S1. Creation of the 14-point CVH score} (DOCX $52 \mathrm{~kb}$ )

Additional file 2: Table S2. Associations between the 14-point CVH score (per 1-unit increase) and the log-transformed subclinical biomarker level stratified by gender. (DOCX $48 \mathrm{~kb}$ )

Additional file 3: Table S3. Associations between the 14-point CVH score (per 1-unit increase) and each component of the subclinical disease markers stratified by gender. (DOCX $51 \mathrm{~kb}$ )

Additional file 4: Table S4. Associations between the 14-point $\mathrm{CVH}$ score (per 1- unit increase) and number of the subclinical disease markers stratified by gender. (DOCX $50 \mathrm{~kb}$ )

\section{Abbreviations \\ ABI: ankle-brachial index; BMI: body mass index; CIMT: carotid intima-media thickness; CRP: C-reactive protein; CVDs: cardiovascular diseases; $\mathrm{CVH}$ : ideal cardiovascular health; DBP: diastolic blood pressure; FPG: fasting plasma glucose; HDL: high-density lipoprotein; IVS: interventricular septum thickness; LDL: Iow-density lipoprotein; Lp-PLA2: lipoprotein-associated phospholipase A2; LV: left ventricular; LVEDD: left ventricular end-diastolic diameter; LVESD: left ventricular end-systolic diameter; LVM: left ventricular mass; PAD: peripheral arterial disease; PW: posterior wall thickness; SBP: systolic blood pressure; SD: standard deviation; TC: total cholesterol}

\section{Acknowledgements}

We thank Chang Liu for his help with the programming and statistical analyses and all the participants for their contributions. 


\section{Availability of data and materials}

The data that support the findings of this study are available from the Third Xiangya Hospital, but restrictions apply concerning the availability of these data, which were used under license for the current study and thus are not publicly available. However, the data are available from the authors upon reasonable request and with permission from the Third Xiangya Hospital.

\section{Authors' contributions}

ZHC conceived and designed the study and is the guarantor. CFW and LZ recruited the participants and collected the data. YQW analysed and interpreted the data and drafted the manuscript. HY and LXW critically revised the manuscript for important intellectual content. All authors read and approved the final manuscript.

\section{Ethics approval and consent to participate}

All participants provided written consent before entering the study, and approval was obtained from the Human Subjects Committee at the Third Xiangya Hospital, Central South University.

\section{Consent for publication}

Not applicable.

\section{Competing interests}

The authors declare that they have no competing interests.

\section{Publisher's Note}

Springer Nature remains neutral with regard to jurisdictional claims in published maps and institutional affiliations.

\section{Author details}

'Department of Health Management Centre, the Third Xiangya Hospital, Central South University, Tongzipo Road 138, Changsha, Hunan Province 410013, China. ${ }^{2}$ Department of General Surgery, the Third Xiangya Hospital, Central South University, Changsha, Hunan Province, China. ${ }^{3}$ Department of Clinical Pharmacology Centre, the Third Xiangya Hospital, Central South University, Changsha, Hunan Province, China. ${ }^{4} Z$ hongguancun Xinzhiyuan Health Management Institute, Beijing, China.

\section{Received: 2 May 2017 Accepted: 10 October 2017}

\section{Published online: 18 October 2017}

\section{References}

1. He J, Gu D, Wu X, Reynolds K, Duan X, Yao C, et al. Major causes of death among men and women in China. N Engl J Med. 2005;353(11):1124-34.

2. Karmali KN, Lloyd-Jones DM. Adding a life-course perspective to cardiovascular-risk communication. Nat Rev Cardiol. 2013;10(2):111-5.

3. Lloyd-Jones DM, Hong Y, Labarthe D, Mozaffarian D, Appel L, Van Horn L, et al. Defining and setting national goals for cardiovascular health promotion and disease reduction: the American Heart Association's strategic impact goal through 2020 and beyond. Circulation. 2010;121(4):586-613.

4. Dong C, Rundek T, Wright CB, Anwar Z, Elkind MS, Sacco RL. Ideal cardiovascular health predicts lower risks of myocardial infarction, stroke, and vascular death across whites, blacks, and hispanics: the northern Manhattan study. Circulation. 2012;125(24):2975-84.

5. Yang Q, Cogswell ME, Flanders WD, Hong Y, Zhang Z, Loustalot F, Gillespie $C$, Merritt R, Trends HFB. In cardiovascular health metrics and associations with all-cause and CVD mortality among US adults. JAMA. 2012;307(12):1273-83.

6. Yang X, Wang A, Liu X, An S, Chen S, Wang Y, Wang Y, Positive WS. Changes in ideal $\mathrm{CVH}$ metrics reduce the incidence of stroke. Sci Rep. 2016;6:19673.

7. Nayor M, Enserro DM, Vasan RS, Xanthakis V. Cardiovascular health status and incidence of heart failure in the Framingham offspring study. Circ Heart Fail. 2016;9(1):e002416.

8. Laitinen TT, Pahkala K, Magnussen CG, Oikonen M, Viikari JS, Sabin MA, et al. Lifetime measures of ideal cardiovascular health and their association with subclinical atherosclerosis: the cardiovascular risk in young Finns study. Int J Cardiol. 2015;185:186-91.

9. Xanthakis V, Enserro DM, Murabito JM, Polak JF, Wollert KC, Januzzi JL, Wang TJ, Tofler G, Vasan RS. Ideal cardiovascular health: associations with biomarkers and subclinical disease and impact on incidence of cardiovascular disease in the Framingham offspring study. Circulation. 2014;130(19):1676-83.

10. Gaye B, Mustafic H, Laurent S, Perier MC, Thomas F, Guibout C, et al. Ideal cardiovascular health and subclinical markers of carotid structure and function: the Paris prospective study III. Arterioscler Thromb Vasc Biol. 2016;36(10):2115-24

11. Tugcu A, Jin Z, Homma S, Elkind MS, Rundek T, Yoshita M, et al. Atherosclerotic plaques in the aortic arch and subclinical cerebrovascular disease. Stroke. 2016:47(11):2813-9.

12. Wang SS, Lay S, Yu HN, Shen SR. Dietary guidelines for Chinese residents (2016): comments and comparisons. J Zhejiang Univ Sci B. 2016:17(9):649-56.

13. Luepker RV, Evans A, McKeigue P, Reddy KS. Cardiovascular survey methods. 3rd ed. Geneva: World Health Organization; 2004.

14. Hypertension EETFftMoA. 2013 practice guidelines for the management of arterial hypertension of the European Society of Hypertension (ESH) and the European Society of Cardiology (ESC): ESH/ESC task force for the Management of Arterial Hypertension. J Hypertens 2013;31(10):1925-1938.

15. Sokolow M, Lyon TP. The ventricular complex in left ventricular hypertrophy as obtained by unipolar precordial and limb leads. Am Heart J. 1949;37(2):161-86

16. Devereux RB, Alonso DR, Lutas EM, Gottlieb GJ, Campo E, Sachs I, Reichek N. Echocardiographic assessment of left ventricular hypertrophy: comparison to necropsy findings. Am J Cardiol. 1986:57(6):450-8.

17. Redlarski G, Palkowski A, Krawczuk M. Body surface area formulae: an alarming ambiguity. Sci Rep. 2016;6:27966.

18. Lang RM, Bierig M, Devereux RB, Flachskampf FA, Foster E, Pellikka PA, et al. Recommendations for chamber quantification: a report from the American Society of Echocardiography's guidelines and standards committee and the chamber quantification writing group, developed in conjunction with the European Association of Echocardiography, a branch of the European Society of Cardiology. J Am Soc Echocardiogr. 2005;18(12):1440-63.

19. Vasan RS, Benjamin EJ, Larson MG, Leip EP, Wang TJ, Wilson PW, Levy D. Plasma natriuretic peptides for community screening for left ventricular hypertrophy and systolic dysfunction: the Framingham heart study. JAMA. 2002;288(10):1252-9.

20. Murabito JM, Evans JC, Nieto K, Larson MG, Levy D, Wilson PW. Prevalence and clinical correlates of peripheral arterial disease in the Framingham offspring study. Am Heart J. 2002;143(6):961-5.

21. Ridker PM, Hennekens CH, Buring JE, Rifai N. C-Reactive protein and other markers of inflammation in the prediction of cardiovascular disease in women. N Engl J Med. 2000;342(12):836-43.

22. Perna AF, Ingrosso D, Lombardi C, Acanfora F, Satta E, Cesare CM, Violetti E, Romano MM, De Santo NG. Possible mechanisms of homocysteine toxicity. Kidney Int Suppl. 2003;84:S137-40.

23. Baszczuk A, Kopczynski Z. Hyperhomocysteinemia in patients with cardiovascular disease. Postepy Hig Med Dosw (Online). 2014;68:579-89.

24. Cerasola G, Cottone S. G. M. The progressive pathway of microalbuminuria: from early marker of renal damage to strong cardiovascular risk predictor. Hypertens. 2010;28(12):2357-69.

25. Blake GJ, Ridker PM. Inflammatory bio-markers and cardiovascular risk prediction. J Intern Med. 2002;252(4):283-94.

26. Clarke R, Stansbie D. Assessment of homocysteine as a cardiovascular risk factor in clinical practice. Ann Clin Biochem. 2001;38(Pt 6):624-32.

27. Lee YY, Yang CK, Weng YM, Chuang CH, Yu W, Chen JC, Li WC. All components of metabolic syndrome are associated with microalbuminuria in a Chinese population. PLoS One. 2016;11(6):e0157303.

28. Blaha MJ, Yeboah J, Al Rifai M, Liu K, Kronmal R, Greenland P. Providing evidence for subclinical CVD in risk assessment. Glob Heart. 2016;11(3):275-85

29. Pahkala K, Hietalampi H, Laitinen TT, Viikari JS, Ronnemaa T, Niinikoski H, et al. Ideal cardiovascular health in adolescence: effect of lifestyle intervention and association with vascular intima-media thickness and elasticity (the special Turku coronary risk factor intervention project for children [STRIP] study). Circulation. 2013;127(21):2088-96.

30. Shah AM, Claggett B, Folsom AR, Lutsey PL, Ballantyne CM, Heiss G, Solomon SD. Ideal cardiovascular health during adult life and cardiovascular structure and function among the elderly. Circulation. 2015;132(21):1979-89.

31. Yan N, Zhou Y, Wang Y, Wang A, Yang X, Russell A, Wu S, Zhao X, Wang W. Association of Ideal Cardiovascular Health and Brachial-Ankle Pulse Wave 
Velocity: a cross-sectional study in northern China. J Stroke Cerebrovasc Dis. 2016;25(1):41-8.

32. Bi $Y$, Jiang $Y$, , He J, Xu Y, Wang $L, X u M$, et al. Status of cardiovascular health in Chinese adults. J Am Coll Cardiol. 2015;65(10):1013-25.

33. Zeng $Q$, Dong SY, Song ZY, Zheng YS, HY W, Mao LN. Ideal cardiovascular health in Chinese urban population. Int J Cardiol. 2013;167(5):2311-7.

34. Shay CM, Ning H, Allen NB, Carnethon MR, Chiuve SE, Greenlund KJ, Daviglus ML, Lloyd-Jones DM. Status of cardiovascular health in US adults: prevalence estimates from the National Health and nutrition examination surveys (NHANES) 2003-2008. Circulation. 2012;125(1):45-56.

35. Hao Z, Zhang Y, Li Y, Zhao J, Zhou Y, Qiu J, Zhao R, Hu J. The association between ideal cardiovascular health metrics and Extracranial carotid artery stenosis in a northern Chinese population: a cross-sectional study. Sci Rep. 2016;6:31720.

36. Coutinho T. Arterial stiffness and its clinical implications in women. Can J Cardiol. 2014;30(7):756-64.

37. Albrektsen G, Heuch I, Lochen ML, Thelle DS, Wilsgaard T, Njolstad I, Bonaa $\mathrm{KH}$. Risk of incident myocardial infarction by gender: interactions with serum lipids, blood pressure and smoking. The Tromso study 1979-2012. Atherosclerosis. 2017;261:52-9.

38. McDermott MM, Liu K, Criqui MH, Ruth K, Goff D, Saad MF, Wu C, Homma S, Sharrett AR. Ankle-brachial index and subclinical cardiac and carotid disease: the multi-ethnic study of atherosclerosis. Am J Epidemiol. 2005;162(1):33-41.

39. Aboyans V, Criqui MH, Abraham P, Allison MA, Creager MA, Diehm C, et al. Measurement and interpretation of the ankle-brachial index: a scientific statement from the American Heart Association. Circulation. 2012;126(24):2890-909.

40. McDermott MM, Applegate WB, Bonds DE, Buford TW, Church T, Espeland MA, et al. Ankle brachial index values, leg symptoms, and functional performance among community-dwelling older men and women in the lifestyle interventions and independence for elders study. J Am Heart Assoc. 2013;2(6):e000257.

41. McDermott MM. Sex differences in the ankle brachial index measurement and interpreting findings of sex differences in peripheral artery disease burden. Circ Cardiovasc Qual Outcomes. 2016;9(2 Suppl 1):S5-7.

\section{Submit your next manuscript to BioMed Central and we will help you at every step:}

- We accept pre-submission inquiries

- Our selector tool helps you to find the most relevant journal

- We provide round the clock customer support

- Convenient online submission

- Thorough peer review

- Inclusion in PubMed and all major indexing services

- Maximum visibility for your research

Submit your manuscript at www.biomedcentral.com/submit 